\title{
Consideraciones taxonómicas y dos nuevas subespecies para Hymenophyllum ferrugineum Colla (Hymenophyllaceae) del Archi- piélago Juan Fernández y del sur de Sudamérica
}

\author{
Taxonomic considerations and two new subspecies for Hymenophyllum ferrugineum Colla \\ (Hymenophyllaceae) from Juan Fernandez Archipelago and southern South America
}

\section{Roberto Rodríguez ${ }^{1}$, Diego Penneckamp ${ }^{2}$ \& Rodrigo Chaura ${ }^{2}$}

${ }^{1}$ Departamento de Botánica, Facultad de Ciencias Naturales y Oceanográficas, Universidad de Concepción, Casilla 160-C, Concepción, Chile.

¿Laboratorio de Biodiversidad y Ecología del Dosel. Facultad de Ciencias Forestales y Recursos Naturales, Universidad Austral de Chile, P.0. Box 567, Valdivia, Chile.

*E-Mail: rrodrigu@udec.cl

\begin{abstract}
A new taxonomic status is proposed for the fern Hymenophyllum ferrugineum, for which two subspecies are recognized based on morphological and biogeography data. One subspecies is recognized as endemic to Juan Fernandez Archipelago and the other endemic to southern South America. It is also clarified the relationship with Hymenophyllum frankliniae, the morphologically closest species native from New Zealand.
\end{abstract}

Hymenophyllum ferrugineum Colla (1836) fue descrito inicialmente sobre la base de material recolectado por Carlo G. Bertero en el Archipiélago Juan Fernández, específicamente en la isla Robinson Crusoe en el año 1830. Basado en las mismas colecciones de Bertero, es descrito Hymenophyllum subtilissimum Kunze (1837); sin embargo, por el Principio III (prioridad) del Código de Nomenclatura Botánica este nombre es considerado inválido. Posteriormente, Hooker (1846) describe Hymenophyllum berteroi, cuyos sintipos corresponden tanto a muestras de Bertero recolectadas en Juan Fernández y a ejemplares provenientes del continente recolectados por $\mathrm{H}$. Cuming en Chiloé.

Por otra parte, material proveniente de Nueva Zelanda fue asignado a $\mathrm{H}$. ferrugineum en Index Filicum de $\mathrm{C}$. Christensen (1906), taxón que aparece como válido y entre los sinónimos están $H$. frankliniae Colenso (1843) y $H$. franklinianum Colenso (1845) de Nueva Zelanda. Este criterio fue seguido por diversos autores en distintos tratamientos taxonómicos y floras donde se cita a $H$. ferrugineum (Christensen \& Skottsberg 1920, Diem \& Lichtenstein 1959, Rodríguez 1995, Rodríguez \& Marticorena 2019).

Iwatsuki (1982) indica diferencias morfológicas que se refiere a los pelos estrellados en su longitud, ramificaciones Open Access Journal

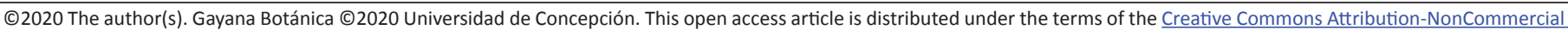
4.0 International License which permits any noncommercial use, distribution, and reproduction, as long as you give appropriate credit to the original author(s) and the source. 
a estas diferencias, indicando además que los pelos del margen de la lámina que son basalmente estrellados y luego tetrafurcados en la gran mayoría de los casos, además los segmentos se mantienen aproximados y paralelos luego de una división, en comparación a los ejemplares continentales de H. ferrugineum y que las plantas de Juan Fernández tenían una mayor relación morfológica con las plantas de Nueva Zelanda, correspondiente a $\mathrm{H}$. frankliniae.

Considerando estas diferencias morfológicas junto con el aislamiento geográfico de las poblaciones insulares relativas a las continentales, aquí proponemos separar a Hymenopyllum ferrugineum en dos subespecies distintas, siendo una endémica del Archipiélago Juan Fernández (Penneckamp 2019) y la otra de los bosques templado lluviosos del sur de Chile y Argentina.
Para el presente trabajo, se empleó una aproximación de taxonomía clásica. Se revisaron ejemplares de los herbarios CONC, SGO y JBN. El material tipo citado fue visto por medio digital desde JSTOR Herbarium o desde los sitios web de los herbarios señalados. Las siglas de los herbarios siguen al Index Herbarorium (Thiers 2019).

\section{TAXONOMÍA}

Descripciones completas de Hymenophyllum ferrugineum (s.l.) se encuentran en Diem \& Lichtenstein (1959), Rodríguez (1995) y Larsen et al. (2013); por lo tanto, se mencionan aquí los caracteres diferenciales que separan a las dos subespecies mediante la siguiente clave:

1. Frondes con las pinnas remotas, subopuestas a alternas. Segmentos pubescentes, con pelos simples, bifurcados hasta estrellados, indusios poco pilosos H. ferrugineum subsp. berteroi 1'. Frondes con las pinnas densas, subopuestas a opuestas, dispuestas hacia el lado acroscópico. Segmentos densamente pubescentes, con pelos basalmente estrellados y luego tetrafurcados, indusios muy pilosos.. H. ferrugineum subsp. ferrugineum

Hymenophyllum ferrugineum Colla subsp. berteroi (Hook.) R.A. Rodr. \& Penneck. comb. et stat. nov.

Basiónimo: Hymenophyllum berteroi Hook. Sp. Fil. 1: 93, lám. 33 C. 1844. (pro parte). (Typus: Chiloé, Cuming n. 11, E00420017! Isotypus: E00215252!)

Sphaerocionium ferrugineum (Colla) Copel., Philipp. J. Sci. 67 (1): 33. 1938. (pro parte)

Hymenophyllum ferrugineum Colla var. donatti Looser, Physis (Buenos Aires) 15: 218, fig. 1. 1939. Typus: Argentina, "Río Negro, Arroyo Frías, 800 m A. Donat 17" (holotipo G00284080).

Sphaerocionium ferrugineum (Colla) Copel. var. donatti (Looser) Kunkel, Nova Hedwigia 9(1-4): 257. 1965.

Iconografía: Larsen et al. 2013: 300, fig. 11; Diem \& Lichtenstein 1959: 737, fig. 26.

Ecología: Especie con amplia distribución, en Chile continental presente desde la Región de la Araucanía hasta la de Magallanes (Mellado-Mansilla et al. 2015, Rodríguez \& Marticorena 2019), con una población de distribución disyunta hacia el norte de su distribución en la Cordillera de
Nahuelbuta, Región del Biobío (Flores 2019). En Argentina se encuentra desde la provincia de Chubut hasta Tierra del Fuego (Zuloaga et al. 2008), desarrollándose de forma epífita sobre troncos y ramas, como también rastrera en sustratos húmedos asociada al estrato muscinal, taludes y paredes rocosas. Especie asociada principalmente a bosques de Nothofagus (Fig. 1).

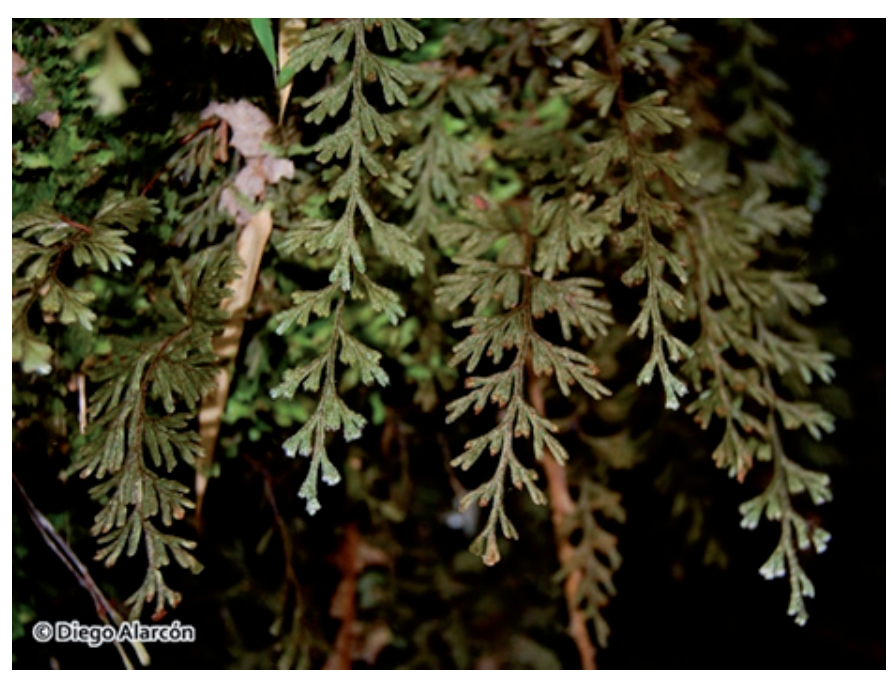

FIGURA 1. Hymenophyllum ferrugineum subsp. berteroi. 


\section{Material estudiado}

CHILE, Región de la Araucanía, Prov. Cautín: Comuna de Pucón, Palguin Alto, 1120 m. 07-I-2015. D. Mellado s.n. (SGO). Región de Los Ríos, Prov. Valdivia: Neltume, Reserva Huilo Huilo, 530 m. I-2011. S. Teillier 6777 (CONC). Región de Los Lagos, Prov. Osorno: Parque Nacional Puyehue, on the way to the Antillanca creater, $4 \mathrm{~km}$ from Aguas Calientes, 04-II-1975. M. Nishida et al. s.n. (SGO). Prov. Llanquihue: Parque Nacional Vicente Pérez Rosales, Cerro Derrumbes, 680-800 m. 23-I-1975. E. Barrera \& M. Rancusi 29 (SGO); Lago Todos los Santos. III- 1974. C. Villagrán (CONC). Prov. Chiloé: Chepu, 16-X-1958. E. J. Godley 214 (SGO); Dalcahue. XII-2010. S. Teillier \& Delaunoy 7149 (CONC); Cordillera de Piuchén, Río Abtao. II-1983. C. Villagrán 4940 (CONC). Prov. Palena: Puerto Chaitén, 27-XI-1943. M. Espinosa s.n, (SGO); Parque Nacional Hornopirén III-1993. R. Godoy et al. 132 (CONC); $8,6 \mathrm{~km} \mathrm{~N}$ of JCT RDS to Chaitén and Palena. 550 m. III-1985. T. Stuessy et al. 7141 (CONC). Región de Aysén, Prov. Aysén: Laguna San Rafael. 100 m. Il-1999. S. Teillier y Márquez 4396 (CONC); Parque Nacional Laguna San Rafael. Istmo de Ofqui. I-1988. E. Pisano 6187 (CONC). Prov. Capitán Prat: Tortel, Isla Berta. 15 m. 02-III-2003. J. Cuvertino s.n. (SGO). Región de Magallanes y de la Antártica Chilena, Prov. Última Esperanza: Puerto Edén, Isla Wellington. XII-1988. E. Pisano 6432 (CONC); Canal Sarmiento, Caleta Ocasión, 31-V-1908. C. Skottsberg 288 (SGO); Puerto Natales, Isla Esmeralda. 20 m. XI-2009. Aravena et al. 612 (CONC). Prov. de Magallanes: Fiordo Nevado, Isla Santa Inés. II-1990. E. Pisano y J. Henríquez 6614 (CONC); Bahía Morris, Isla Capitán Aracena. XII-1971. E. Pisano 3333 (CONC).

\section{Hymenophyllum ferrugineum Colla subsp. ferrugineum}

Herb. Pedem. 6: 190. 1836. "Ad rupes et arborum radices in sylvis umbrosis montium editiorum ins. Juan Fernandez Bertero 1540". Typus: Bertero 1540 (Lectotipo aquí designado: P00622062!; Isolectotipos: GH00021351!, MPU018007!, MPU018008!, P00622060!, P00622061!, US00067522!)

Hymenophyllum subtilissimum Kunze, Analecta Pteridogr. 49. 1837. "Juan Fernández, Bertero 1540".

Sphaerocionium ferrugineum (Colla) Copel., Philipp. J. Sci. 67 (1): 33. 1938. (pro parte)

Iconografía: Johow, Anales Univ. Chile 82: fig. 31. 1893.

Ecología: Endemismo del Archipiélago Juan Fernández. Especie típica de la mirtisilva de altitud (Danton 2006) o "upper montane forest" (Skottsberg 1953) de Robinson Crusoe y Alejandro Selkirk, encontrándose por sobre los 450 m s.m. en bosques densos y con nieblas frecuentes, donde se desarrolla en condiciones sombrías en taludes húmedos, estrato muscinal y epífito en bases de troncos y raíces (Penneckamp 2018) (Fig. 2).

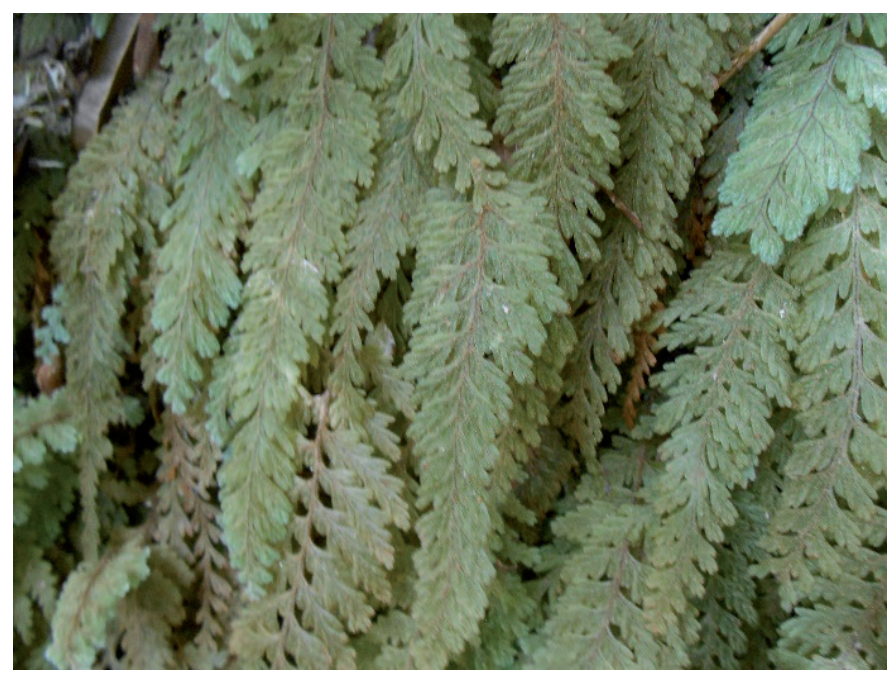

Figura 2. Hymenophyllum ferrugineum subsp. ferrugineum. Fotografiado en el Mirador de Selkirk, Isla Robinson Crusoe.

Material estudiado

CHILE, Región de Valparaíso, Prov. Valparaíso, Archipiélago Juan Fernández, Isla Robinson Crusoe (Más a Tierra): Quebrada. Piedra Agujereada, 590 m. 26-VII-1990. M. Ricci s.n. (JBN); Cerca del Mirador de Selkirk, 470 m. 28-XI-1983. E. Barrera s.n. (SGO); Cordón Chifladores, 660 m. 08-XII1916. C. \& I. Skottsberg 76 (SGO); Mirador de Selkirk, 575 m. I-1980. C. Marticorena et al. 9033 (CONC).

Isla Alejandro Selkirk (Más Afuera): Quebrada de Las Chozas, 700 m. 03-III-1917. C. \& I. Skottsberg 535 (SGO); Quebrada Chorro de Doña María. 850 m. II-1986. H. Valdebenito 9606 A (CONC); Cuchillo del Imán. I-1986. A. Landero y Gaete 9172 (CONC); Sendero Monte de Quebrada Vacas-Las Torres, 690 m. II-1980. T. Stuessy et al. 5077 (CONC).

\section{AGRADECIMIENTOS}

Gloria Rojas, Museo Nacional de Historia Natural (SGO), Alicia Marticorena, Herbario de la Universidad de Concepción (CONC) y Diego Reyes, Jardín Botánico Nacional, por la facilitación de datos. Carlos Lehnebach y Leon Perrie del Museum of New Zealand Te Papa Tongarewa, por sus comentarios acerca de $\mathrm{H}$. frankliniae y a Atsushi Ebihara del National Museum of Nature and Science de Japón por el 
envío de bibliografía. Agradecemos al Dr. Diego Alarcón por su voluntad de ceder el uso de su fotografía de $H$. ferrugineum subsp. berteroi para esta publicación.

\section{REFERENCIAS}

Brownsey, P.J., Perrie, L.R. 2016. Taxonomic notes on the New Zealand flora: lectotypes in the fern family Hymenophyllaceae. New Zealand Journal of Botany 54(1): 48-62.

Christensen, C. 1906. Index Filicum, sive enumeration omnium generum specierumque. Hafniae. 744 pp.

Christensen, C., Skottsberg, C. 1920. The Pteridophyta of Juan Fernandez Islands. In: Skottsberg, C. (Ed.) Natural History of Juan Fernandez and Easter Island, Vol. 2, pp. 1-46. Botany. Almqvist \& Wiksells Eds., Uppsala, Sweden.

Colenso, W. 1843. Description of some new ferns lately discovered in New Zealand. Tasmanian Journal of Natural Science 1: 375-379.

Colenso, W. 1845. A classification and description of some newly discovered ferns, collected in the Northern Island of New Zealand, in the summer of 1841-2. Tasmanian Journal of Natural Science 2: 161-189.

Colla, L.A. 1836. Herbarium Pedemontanum juxta methodum naturalem dispositum additis stirpibus exoticis ad universos ejusdem method ordines exhibendos. Torino. Vol. 6.

Danton, P. 2006. La "myrtisylve" de l'archipel Juan Fernández (Chile), une forêt en voie de disparation rapide. Acta Botanica Gallica 153: 179-199.

Diem, J., Lichtenstein, J.S. 1959. Las Hymenofiláceas del área argentino-chilena del sud. Darwiniana 11(4): 633-760.

Ebihara, A., Dubuisson, J.-Y., Iwatsuki, K., Hennequin, S., Ito, M. 2006. A taxonomic revision of Hymenophyllaceae. Blumea-Biodiversity, Evolution and Biogeography of Plants 51(2): 221-280.

Ebihara, A., Hennequin, S., Iwatsuki, K., Bostock, P.D., Matsumoto, S., Jaman, R., Dubuisson, J-Y., Dubuisson, M. 2004. Polyphyletic origin of Microtrichomanes (Prantl) Copel. (Hymenophyllaceae), with a revision of the species. Taxon 53(4): 935-948.

Ebihara, A., Nitta, J.H., Iwatsuki, K. 2010. The Hymenophyllaceae of the Pacific area. 2. Hymenophyllum (excluding subgen. Hymenophyllum). Bulletin of the National Science Museum, series B (Botany) 36: 43-59.

Flores, E. 2019. Nuevo rango de distribución de Hymenophyllum ferrugineum Colla (Hymenophyllaceae) en Chile continental. Boletín Nahuelbuta Natural 4: 3.

Hennequin, S., Ebihara, A., Dubuisson, J.-Y., Schneider, H. 2010. Chromosome number evolution in Hymenophyllum (Hymenophyllaceae), with special reference to the subgenus Hymenophyllum. Molecular Phylogenetic and Evolution 55: 47-59.

Hooker, W.J. 1844-1846: Species Filicum. Vol. 1. Pamplin, London.

Iwatsuki, K. 1982. Studies in the systematic of filmy ferns VI. The genus Sphaerocionium in Asia and Oceania. Journal of the Faculty of Science, University of Tokyo, Section III, Botany 13: 203-215.

Larsen, C., Ponce, M.M., Scataglini, M.A. 2013. Revisión de las especies de Hymenophyllum (Hymenophyllaceae) del sur de Argentina y Chile. Gayana Botánica 70(2): 274-329.

Mellado-Mansilla, D., Díaz, I., Moreno, R. 2015. Ampliación del rango de distribución de Hymenophyllum ferrugineum Colla (Hymenophyllaceae) para Chile continental. Chloris Chilensis (18)1.

Penneckamp, D. 2018. Flora Vascular Silvestre del Archipiélago Juan Fernández. Primera Edición (versión electrónica). Planeta de Papel Ediciones, Valparaíso, Chile. 723 pp.

Penneckamp, D. 2019. Suplemento a la Flora Vascular Silvestre del Archipiélago Juan Fernández. Capítulo adicional: 724-750 pp.

Rodríguez, R. 1995. Pteridophyta. En: Marticorena, C., Rodríguez, R. (Eds.) Flora de Chile, Vol. 1, pp. 119-309. Ediciones Universidad de Concepción, Concepción, Chile.

Rodríguez, R., Marticorena, A. (Eds.). 2019. Catálogo de las plantas vasculares de Chile. Editorial Universidad de Concepción, Chile. 420 pp.

Skottsberg, C. 1953. The vegetation of the Juan Fernandez Islands. In: Skottsberg, C. (Ed.) Natural History of Juan Fernandez and Easter Island, Vol. 2, 793-960 pp + planches 58-116. Botany. Almqvist \& Wiksells Eds., Uppsala, Sweden.

Thiers, B. 2019 (continuously updated). Index Herbariorum: a global directory of public herbaria and associated staff. New York Botanical Garden's Virtual Herbarium. Available from: http://sweetgum.nybg.org/science/ih/ Accessed: April 27, 2020.

Zuloaga, F.O., Morrone, O., Belgrano, M.J., Marticorena, C., Marchesi, E. (Eds.). 2008. Catálogo de las Plantas Vasculares del Cono Sur (Argentina, Sur de Brasil, Chile, Paraguay y Uruguay. Monographs in Systematic Botany from the Missouri Botanical Garden 107 (3 vols.): i-xcvi. 3348 pp. 